Structural equation modelling proved a useful method for exploring and quantifying realist theory. The analysis was limited by available data; therefore future research would benefit from primary data collection.

\section{P32 EXPERIENCES OF WOMEN FROM A LOWER SOCIOECONOMIC BACKGROUND WHEN USING HEALTHY EATING MOBILE APPS: A QUALITATIVE INTERVIEW STUDY}

${ }^{1}$ SJ Flaherty ${ }^{*},{ }^{1,2} \mathrm{M}$ McCarthy, ${ }^{3} \mathrm{~A}$ Collins, ${ }^{1,4} \mathrm{~F}$ McAuliffe. ${ }^{1} \mathrm{HRB}$ Centre for Health and Diet Research, University College Cork, Cork, Ireland; ${ }^{2}$ Cork University Business School, University College Cork, Cork, Ireland; ${ }^{3}$ Department of Food Business and Development, University College Cork, Cork, Ireland; ${ }^{4}$ Obstetrics and Gynaecology, School of Medicine, University College Dublin, Dublin, Ireland

\subsection{6/jech-2017-SSMAbstracts. 134}

Background Mobile apps offer a potentially effective approach to support healthier food behaviours if adequately designed and informed by behaviour change theory. Individuals from a lower socioeconomic background often report unhealthier dietary patterns and consequently may benefit from a mobile app intervention supporting healthier food behaviours. However, there is limited evidence available on the use of mobile health apps in this group. Previous work suggests that a reasonable standard of health and nutrition literacy is required for effective use of existing healthy eating mobile apps but this knowledge is often low in those from a lower socioeconomic background. Consequently, it is unclear if existing mobile apps are appropriate for this population group. The aim of this study is to explore the experiences of women from a lower socioeconomic background when using healthy eating mobile apps and the individual-level and mobile-specific factors that influence their experiences.

Methods A purposive sample of 15 women from a lower socioeconomic background and aged between 18-50 years were selected to participate. Participants completed a questionnaire assessing nutrition knowledge before using the assigned mobile apps. A total of three mobile apps were assessed in this study and were of varying quality in relation to nutrition content, behaviour change and user quality. Each participant was assigned to use two different mobile apps and used each for one week only. Assignment order was randomised. After the two-week period, semi-structured interviews were conducted with participants to discuss their experiences. Interviews were audio-recorded, transcribed verbatim, and analysed using a thematic analysis approach.

Results Preliminary analysis suggests that overall mobile app quality is adequate but there is a need to improve the customisability of mobile apps to ensure they fit users' needs. The food lives of participants vary and mobile apps need to be flexible to reflect this variety for integration of mobile apps into everyday life. The language used in a mobile app was a reason for discontinuing use as it was not clearly understood by users or was viewed as irrelevant.

Conclusion Existing mobile apps may support healthier food behaviours in women from a lower socioeconomic background but changes in design may be required. A user-centred approach is recommended where users from a lower socioeconomic background are engaged at all stages of the design process. This may improve their relevance to this population group and increase their effectiveness in supporting healthier food behaviours.

\section{P33 WORKING UPSTREAM: EXAMINING A CENTRAL IDEA IN ADDRESSING HEALTH INEQUALITIES}

${ }^{1} \mathrm{NE}$ McMahon*, ${ }^{2} \mathrm{M}$ Gabbay, ${ }^{3} \mathrm{~J}$ Jagosh, ${ }^{1} \mathrm{CL}$ Watkins. ${ }^{1}$ Faculty of Health and Wellbeing, University of Central Lancashire, Preston, UK; ${ }^{2}$ Institute of Health and Society, University of Liverpool, Liverpool, UK; ${ }^{3}$ Centre for Advancement in Realist Evaluation and Synthesis, University of Liverpool, Liverpool, UK

\subsection{6/jech-2017-SSMAbstracts. 135}

Background Health inequalities are variations or differences in health that are systematic, socially produced and unfair. Despite an improved understanding of the causes of health inequalities, there is an overreliance on individual level interventions, often in the form of behaviour change or lifestyle interventions. It is increasingly recognised that in order to reduce health inequalities, there is a need to engage in more 'upstream' action. However, as action to reduce health inequalities becomes progressively more interdisciplinary, it is unclear the extent to which there is consensus as to what constitutes 'upstream' working to reduce health inequalities.

Methods The aim of this study is to examine interdisciplinary conceptualisations of upstream action to reduce health inequalities. Conceptualisations of upstream action have been identified through (i) a review of the literature and (ii) semistructured interviews with researchers, practitioners and members of the public actively involved in research and practice to reduce health inequalities in the North West of England. This study is being undertaken as part of an MPhil/PhD study. Interviews and data analysis are ongoing.

Results There exist multiple different, but related, conceptualisations of upstream action. Examples include upstream action as any intervention focusing on prevention; 'low agency' or 'population' interventions; action on the social determinants of health; and grass roots or bottom up action that involves shifting the balance of power from individuals making decisions to individuals who are affected by decisions. There is a distinction that can be made between 'upstream determinants' of health and health inequalities, and 'upstream action', whereby people or interventions located 'downstream' can act to influence more upstream determinants of health inequalities.

Conclusion There is a need to further unpack, and clarify, what we understand by 'upstream action', and the ways in which such actions produce their effects, to assist researchers and practitioners to operationalise and embed these seminal ideas into their day to day practice.

\section{P34 EXPLAINING DIFFERENCES IN CARDIOVASCULAR DISEASE MORTALITY BETWEEN LOCAL AUTHORITIES IN ENGLAND}

P Bhatnagar*, N Townsend. Nuffield Department of Population Health, University of Oxford, Oxford

\subsection{6/jech-2017-SSMAbstracts. 136}

Background Substantial inequalities in age-standardised cardiovascular disease (CVD) mortality rates exist at the local authority (LA) level within England, with particular areas having consistently higher rates. Higher deprivation is associated with higher CVD mortality, but we know little about how the demographics and environments of LAs contribute to variations in mortality rates. Our aim was to explore the extent to which demographic, behavioural and environmental factors 